Acknowledgements: We would like to thank Andrea Baker, English mother tongue speaker, whose work has been very helpful in editing this paper.

\title{
References
}

1 Pai M, Denkinger CM, Kik SV, et al. Gamma interferon release assays for detection of Mycobacterium tuberculosis infection. Clin Microbiol Rev 2014; 27: 3-20.

2 Diel R, Goletti D, Ferrara G, et al. Interferon- $\gamma$ release assays for the diagnosis of latent Mycobacterium tuberculosis infection: a systematic review and meta-analysis. Eur Respir J 2011; 37: 88-99.

3 Sester M, van Leth F, Bruchfeld J, et al. Risk assessment of tuberculosis in immunocompromised patients. A TBNET study. Am J Respir Crit Care Med 2014; 190: 1168-1176.

4 Sañé Schepisi M, Parracino MP, Mammone A, et al. Immune status and serial Quantiferon-TB Gold In-tube screening for latent Mycobacterium tuberculosis infection among HIV-infected persons in a country with a low tuberculosis incidence. J Infect Dis 2015; 211: 1852-1853.

5 Smith R, Cattamanchi A, Steingart KR, et al. Interferon- $\gamma$ release assays for diagnosis of latent tuberculosis infection: evidence in immune-mediated inflammatory disorders. Curr Opin Rheumatol 2011; 23: 377-384.

6 Denkinger CM, Dheda K, Pai M. Guidelines on interferon- $\gamma$ release assays for tuberculosis infection: concordance, discordance or confusion? Clin Microbiol Infect 2011; 17: 806-814.

7 Bartalesi F, Vicidomini S, Goletti D, et al. QuantiFERON-TB Gold and the TST are both useful for latent tuberculosis infection screening in autoimmune diseases. Eur Respir J 2009; 33: 586-593.

8 European Centre for Disease Prevention and Control. Use of interferon-gamma release assays in support of TB diagnosis. Stockholm, ECDC, 2011.

9 Gardam MA, Keystone EC, Menzies R, et al. Anti-tumour necrosis factor agents and tuberculosis risk: mechanisms of action and clinical management. Lancet Infect Dis 2003; 3: 148-155.

10 Cellestis. QuantiFERON -TB Gold. http://www.quantiferon.com/IRM/pub/ErrorPageNotFound.aspx?aspxerrorpath=/ $\mathrm{irm} /$ content/quantiferon-tb-gold1.aspx Date last accessed: December 1, 2014.

11 OxfordImmunotec. T.SPOT1.TB. www.oxfordimmunotec.com/T-SPOT.TB_International Date last accessed: December 1, 2014.

12 Fliess JL. The measurement of inter-rate agreement. In: Bradley RA, Levin B, Cho Paik M, eds. Statistical methods for rates and proportions. New York, John Wiley \& Sons Inc, 1981; pp. 212-236.

13 Farhat M, Greenaway C, Pai M, et al. False-positive tuberculin skin tests: what is the absolute effect of BCG and non-tuberculous mycobacteria? Int J Tuberc Lung Dis 2006; 10: 1192-1204.

14 Tagmouti S, Slater M, Benedetti A, et al. Reproducibility of interferon gamma release assays: a systematic review. Ann Am Thorac Soc 2014; 11: 1267-1276.

15 Mack U, Migliori GB, Sester M, et al. LTBI: latent tuberculosis infection or lasting immune responses to M. tuberculosis? A TBNET consensus statement. Eur Respir J 2009; 33: 956-973.

\section{Phenotyping chronic pulmonary aspergillosis by cluster analysis}

To the Editor:

Chronic pulmonary aspergillosis (CPA) is a complex disorder involving various underlying conditions and risk factors, clinical and radiological features, and natural histories or responses to treatment [1]. Untreated, patients with CPA have $\geqslant 50 \% 5$-year mortality $[2,3]$. Recently, it was proposed that CPA includes simple aspergilloma, chronic cavitary pulmonary aspergillosis (CCPA) and chronic necrotising pulmonary aspergillosis (CNPA) [1].

However, aside from simple aspergilloma, the presentation and treatment of which is distinct from that of CCPA and CNPA, considerable overlap seems to exist between the various clinical, radiological and histological presentations of CCPA and CNPA $[1,4]$. Improving the classification of CCPA and CNPA by integrating the multiple aspects of the disease is critical for future clinical trials. Here, we used a subject-centred multivariate clustering approach without a priori assumptions to identify phenotypes among patients with CPA (excluding simple aspergilloma) based on integrated clinical, biological and radiological features.

This study was based on a retrospective analysis of 127 patients with CPA (simple aspergilloma excluded) seen between January 2002 and December 2011 in eight chest departments at French university hospitals. The study was approved by the local research Ethics Committee (DR-2012-304) of Poitiers, France. 
CPA was diagnosed according to the following criteria (all criteria were required) [1]. 1) Pulmonary cavitation(s) with a wall and possible pleural thickening on imaging with or without intracavitary mass or other radiological patterns compatible with CPA, such as progressive consolidation with or without secondary cavitation. 2) Serological or microbiological evidence implicating Aspergillus spp. 3) The presence of persistently elevated inflammatory markers. 4) Exclusion of all other causes with a similar disease presentation (e.g. active tuberculosis, nontuberculous mycobacterial infection and other bacterial infections). Subjects were required to have had a whole chest computed tomography (CT) at the time of diagnosis. Exclusion criteria were simple aspergilloma, invasive aspergillosis, allergic bronchopulmonary aspergillosis and significant immunosuppression (uncontrolled HIV, haematological malignancy, chronic granulomatous disease or glucocorticoid dose $>7.5 \mathrm{mg}$ of prednisolone per day).

Demographic, clinical and radiological data were collected at the time of diagnosis. CT images were reviewed by two chest radiologists blinded to the clinical data (F. Laurent and C. Beigelman-Aubry), who reached a final decision by consensus. The following features were collected in each lung: number/volume of cavities, maximal cavity wall thickness, number/volume of fungus balls, and maximal pleural thickness. Alveolar consolidation, lobar collapse(s), tree-in-bud and nodules $>5 \mathrm{~mm}$ were visually quantified. The presence, severity and extent of bronchiectasis, and bronchial wall thickening were assessed [5].

A hierarchical ascendant classification [6] was used to identify relevant subgroups without prior assumptions [7]. Importantly, the classical subtypes of CCPA and CNPA used in previous classifications were not included in the analysis. The following characteristics were included [1]: age; body mass index (BMI); sex; chronic obstructive pulmonary disease; prior tuberculosis, nontuberculous mycobacterial infection, sarcoidosis, interstitial pneumonia or thoracic surgery; prior glucocorticoid and antifungal treatments; alcoholism; diabetes; smoking habit; the presence of fever, chest pain, haemoptysis or weight loss; the time frame of the evolution of symptoms (1-3 months versus $>3$ months); the presence of cough, dyspnoea and expectoration; precipitating (IgG) antibodies to Aspergillus (Microgen Bioproduct Ltd, Camberley, UK) in serum; Aspergillus cultures; C-reactive-protein; serum galactomannan antigen; the presence of cavity, fungus balls, collapse, tree-in-bud, nodules $>5 \mathrm{~mm}$ and areas of consolidation; bronchiectasis CT score; and the maximum thickness of the cavity wall and pleura. All quantitative and qualitative variables were discretized using a multiple correspondence analysis for standardisation before cluster analysis.

Out of the 127 patients, 106 were assessed for the cluster analysis (14 patients had non-usable CT and seven were excluded due to missing clinical data). 70 (66.0\%) were male, with a median age of 58 years (range: 46-66 years), and a median BMI of $20.0 \mathrm{~kg} \cdot \mathrm{m}^{-2}$ (range: $17.5-22.5 \mathrm{~kg} \cdot \mathrm{m}^{-2}$ ). The most common underlying conditions were previous history of tuberculosis (49.1\%) and chronic obstructive pulmonary disease (34\%). More than two thirds of the patients had underlying risk factors, mainly smoking (44.3\%), glucocorticoid use (36.8\%) and alcoholism (21.7\%). The commonest presenting symptoms were cough (86.8\%), sputum (75.5\%), haemoptysis (48.1\%) and weight loss (51\%). Direct examination of sputum revealed Aspergillus hyphae in $38(35.8 \%)$ cases. Sputum cultures were positive in $62.3 \%$ of cases, with positive serum galactomannan antigen in eight (18\%) out of 45 cases. Aspergillus fumigatus precipitins were positive in $85 \%$ of cases. The most common CT abnormalities were the presence of cavities (86.8\%) with thick walls (85\%), adjacent pleural thickening (81.6\%) and fungus balls (56.6\%). Additional features were predominantly areas of consolidation (68\%), lobar collapse (64.1\%) and nodules $>5 \mathrm{~mm}$ (37.7\%). Bronchiectasis was present in $78 \%$ of cases, and was mild in $27 \%$ of cases, moderate in $46 \%$ and severe in $19 \%$.

No significant differences were found between radiological and clinical variables of the patients included in the cluster analysis and those of patients not eligible for the analysis. Only one phenotype was identified according to the clustering approach. The dendrogram (fig. 1) showed the distances between groups to be small, expressing little dissimilarity between them. According to the level of clustering, we nevertheless considered two and three potential subgroups, with very few patients (six versus 100 patients and four versus two versus 100 patients, respectively), and explored their relevance. Secondary analysis of these groups did not show relevant clinical or imaging characteristics. Hence, the 106 patients with CPA were considered relatively homogenous with regard to the epidemiological, clinical, and radiological features at diagnosis. Notably, the classical subtypes of CPA, i.e. CNPA or CCPA, were not identified by the cluster analysis.

The CNPA criteria originally defined by BINDER et al. [8] and then by GEFTER et al. [9] are not strictly equivalent to those proposed by DeNNING et al. [1] more recently. BINDER et al. [8] suggested a definition of CNPA involving histological evidence. While GEFTER et al. [9] proposed a radiological definition based on the presence of a pre-existing cavity to distinguish the invasive and noninvasive forms. The criteria they used to distinguish CCPA and CNPA are neither specific nor based on a large prospective series with supporting histological evidence. All three definitions include the notion of baseline imaging before more invasive investigations are considered, and histological data are rarely available. Recently, IzumikAwA et al. [10] retrospectively studied 27 cases of $\mathrm{CPA}$, and divided them into four groups according to the presence of 


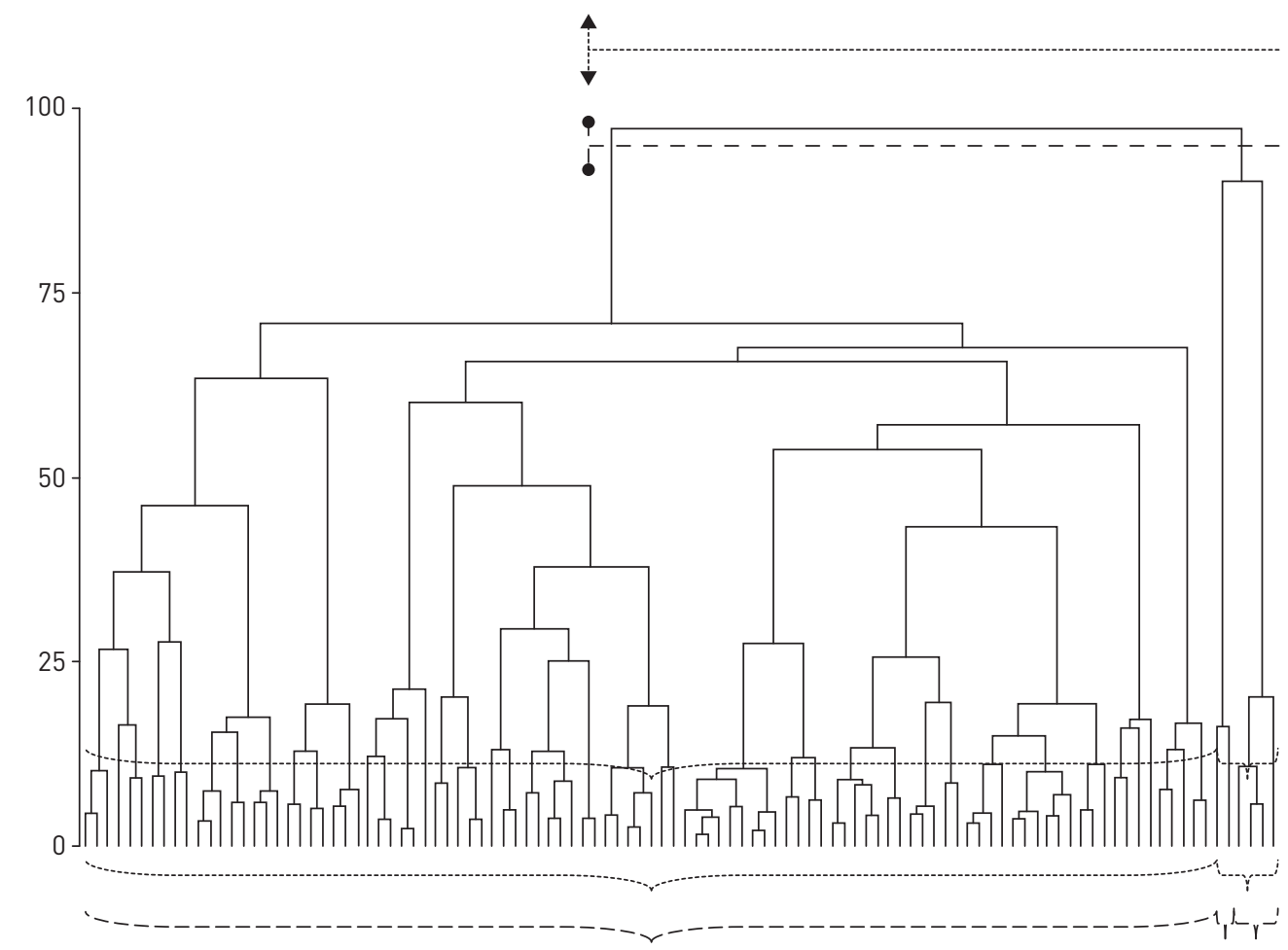

FIGURE 1. Dendrogram based on the characteristics of patients at the time of diagnosis $(n=106)$. The dotted line, arrows and curly brackets show the results of clustering after the first division, while the dashed symbols show the results of clustering after two divisions. The $x$-axis represents patients classified according to the clusters to which they belong and the y-axis represents distance reflecting the dissimilarity between the two clusters.

histological lung invasion, in order to determine whether clinical or radiological parameters could discern between CCPA and CNPA. Being unable to distinguish CCPA from CNPA based on clinical and radiological settings, these authors have proposed the term "chronic progressive pulmonary aspergillosis" for a broad clinical syndrome including both CCPA and CNPA. Our study in a large series of CPA patients was consistent with the findings of IzUMIKAWA et al. [10]. Furthermore, the two classical subtypes of CCPA and CNPA did not emerge as two separate clusters. Limitations of our study included the retrospective design and the absence of histological data, as they are rarely available in clinical practice. The hierarchical ascendant classification [6] is, by nature, exploratory and aims at revealing patterns without any a priori assumptions; however, it does not allow estimation of the study power. The main strength of the study is to provide, in the largest published series, to date, of a still poorly known pulmonary disease, a rigorous CT assessment with a thorough clinical and biological evaluation.

In conclusion, our data demonstrate that patients with CPA (excluding simple aspergilloma) have relatively homogeneous characteristics, and support the notion of a continuum in the clinical and radiological features of CPA rather than identifying somewhat artificial subtypes as classically proposed.

0 $@$ ERSpublications

Cluster analysis based on clinical and radiological settings does not distinguish any specific phenotype of CPA http://ow.ly/QZq7V

Cendrine Godet ${ }^{1}$, François Laurent ${ }^{2,3}$, Guillaume Béraud ${ }^{1,4,5}$, Cécile Toper ${ }^{6}$, Boubou Camara ${ }^{7}$, Bruno Philippe ${ }^{8}$, Patrick Germaud ${ }^{9}$, Vincent Cottin ${ }^{10,11}$, Catherine Beigelman-Aubry ${ }^{12}$, Antoine Khalil ${ }^{13}$, Pascal Blouin ${ }^{14}$, Mathilde Pouriel $^{15}$, France Roblot ${ }^{1,16,17}$, Anne Bergeron ${ }^{18,19}$ and Jacques Cadranel ${ }^{6,20}$ for the ACHROSCAN study group $^{21}$

${ }^{1}$ Service de Maladies Infectieuses et Tropicales, CHU Poitiers, Poitiers, France. ${ }^{2}$ Service d'Imagerie médicale, radiologie diagnostique et thérapeutique, CHU de Bordeaux, Bordeaux, France. ${ }^{3}$ Université Bordeaux, Centre de Recherche Cardio-Thoracique, INSERM U1045, Bordeaux, France. ${ }^{4}$ EA2694, Université Droit et Santé Lille 2, Lille, France. ${ }^{5}$ Interuniversity Institute for Biostatistics and Statistical Bioinformatics, Hasselt University, Hasselt, Belgium. ${ }^{6}$ Service de Pneumologie, AP-HP, Hôpital Tenon et Université P\&M Curie, Paris, France. ${ }^{7}$ Clinique Universitaire de Pneumologie, Pôle Thorax et Vaisseaux, CHU de Grenoble, Grenoble, France. ${ }^{8}$ Service de Pneumologie, Hôpital René Dubos, Pontoise, France. ${ }^{9}$ Service de Pneumologie, CHU Nantes, Nantes, France. ${ }^{10}$ Centre National de référence des maladies pulmonaires rares, Service de Pneumologie, CHU Lyon hôpital Louis Pradel, Lyon, France. ${ }^{11}$ Université Claude Bernard 
Lyon 1, UMR754, Lyon, France. ${ }^{12}$ Service de radiodiagnostic et radiologie interventionnelle CHUV, Lausanne, Switzerland. ${ }^{13}$ Service de Radiologie, AP-HP, Hôpital Tenon, Paris, France. ${ }^{14}$ Direction de la Recherche clinique et de l'innovation, CHU Poitiers, Poitiers, France. ${ }^{15}$ Kappa Santé, Paris, France. ${ }^{16}$ Faculté de médecine et de Pharmacie, Université de Poitiers, Poitiers, France. ${ }^{17}$ U1070 Inserm, Poitiers, France. ${ }^{18}$ AP-HP, Hôpital Saint-Louis, Service de Pneumologie, Paris, France. ${ }^{19}$ Université Paris Diderot, Sorbonne Paris Cité, U1153 CRESS, Biostatistics and Clinical Epidemiology research team, Paris, France. ${ }^{20}$ Sorbonne Université, UPMC Université Paris 06, Paris, France. ${ }^{21} \mathrm{~A}$ full list of the ACHROSCAN (CT-scan evaluation of chronic pulmonary aspergillosis) study group members and their affiliations can be found in the Acknowledgements section.

Correspondence: Cendrine Godet, Service des Maladies Infectieuses et Tropicales, CHU la Milétrie, 2 rue de la Milétrie, 86021 Poitiers cedex, France. E-mail: c.godet@chu-poitiers.fr

Received: April 052015 | Accepted after revision: July 062015 | First published online: Sept 172015

Support statement: Financial support was provided by the following organisations: Pfizer, Paris, France; ASTELLAS Pharma SAS, France; SOS Oxygène, Nice, France; ISIS Médical, France; AADAIRC, Poitou-Charentes, France. Funding information for this article has been deposited with FundRef.

Conflict of interest: Disclosures can be found alongside the online version of this article at erj.ersjournals.com

Acknowledgements: Philippe Gegou (INTRASENSE technical and computer support team, Montpellier, France) who enabled us to work on Myrian, the software suite used for the visualisation and post-processing of our medical images.

The authors also wish to thank Jeffrey Arsham (an American medical translator working at CHU Poitiers, Poitiers, France) for reviewing and editing the original English language manuscript. Marie France Carette (Service de Radiologie, AP-HP, Hôpital Tenon, Paris, France) and Gilbert Ferriti (Service de Radiologie, CHU Grenoble, Grenoble, France) both radiologists who assisted us with data collection. Jean-Pierre Frat (Service de Réanimation médicale, CHU Poitiers, Poitiers, France) for editing the original manuscript.

The following investigators participated in the ACHROSCAN study group: François Laurent, CHU Bordeaux, Bordeaux, France; Boubou Camara and Christophe Pison, CHU Grenoble, Grenoble, France; Catherine Beigelman-Aubry, CHUV Lausanne, Lausanne, Switzerland; Vincent Cottin CHU Lyon, Lyon, France; Patrick Germaud, CHU Nantes, Nantes, France; Anne Bergeron, APHP Paris, Paris, France; Jacques Cadranel, Antoine Khalil and Cécile Toper, APHP, Paris, France; Guillaume Béraud, Pascal Blouin and Cendrine Godet CHU Poitiers, Poitiers, France; Bruno Philippe, $\mathrm{CH}$ Pontoise, Pontoise, France.

Cendrine Godet, Jacques Cadranel and Anne Bergeron are members of the GREPI (French Group for Research and Education in Respiratory Infectious Diseases).

\section{References}

1 Denning DW, Riniotis K, Dobrashian R, et al. Chronic cavitary and fibrosing pulmonary and pleural aspergillosis: case series, proposed nomenclature change, and review. Clin Infect Dis 2003; 37: Suppl 3, S265-S280.

2 Nam HS, Jeon K, Um SW, et al. Clinical characteristics and treatment outcomes of chronic necrotizing pulmonary aspergillosis: a review of 43 cases. Int J Infect Dis 2010; 14: e479-e482.

3 Ohba H, Miwa S, Shirai M, et al. Clinical characteristics and prognosis of chronic pulmonary aspergillosis. Respir Med 2012; 106: 724-729.

4 Agarwal R, Vishwanath G, Aggarwal AN, et al. Itraconazole in chronic cavitary pulmonary aspergillosis: a randomised controlled trial and systematic review of literature. Mycoses 2013; 56: 559-570.

5 Bhalla M, Turcios N, Aponte V, et al. Cystic fibrosis: scoring system with thin-section CT. Radiology 1991; 179: 783-788.

6 Burgel PR, Paillasseur JL, Caillaud D, et al. Clinical COPD phenotypes: a novel approach using principal component and cluster analyses. Eur Respir J 2010; 36: 531-539.

7 Wardlaw AJ, Silverman M, Siva R, et al. Multi-dimensional phenotyping: towards a new taxonomy for airway disease. Clin Exp Allergy 2005; 35: 1254-1262.

8 Binder RE, Faling LJ, Pugatch RD, et al. Chronic necrotizing pulmonary aspergillosis: a discrete clinical entity. Medicine (Baltimore) 1982; 61: 109-124.

9 Gefter WB, Weingrad TR, Epstein DM, et al. "Semi-invasive" pulmonary aspergillosis: a new look at the spectrum of aspergillus infections of the lung. Radiology 1981; 140: 313-321.

10 Izumikawa K, Tashiro T, Tashiro M, et al. Pathogenesis and clinical features of chronic pulmonary aspergillosis is it possible to distinguish CNPA and CCPA clinically? J Infect Chemother 2014; 20: 208-212. 